\title{
Ratio Comparisons of Supremum and Stop Rule Expectations
}

\author{
Theodore P. Hill and Robert P. Kertz
}

Georgia Institute of Technology, Dept. of Mathematics, Atlanta, Georgia 30332, USA

Summary. Suppose $X_{1}, X_{2}, \ldots, X_{n}$ are independent non-negative random variables with finite positive expectations. Let $T_{n}$ denote the stop rules for $X_{1}, \ldots, X_{n}$. The main result of this paper is that $E\left(\max \left\{X_{1}, \ldots, X_{n}\right\}\right)$ $<2 \sup \left\{E X_{t}: t \in T_{n}\right\}$. The proof given is constructive, and sharpens the corresponding weak inequalities of Krengel and Sucheston and of Garling.

\section{$\S 1$. Introduction}

Let $X_{1}, X_{2}, \ldots, X_{n}$ be independent non-negative random variables on a probability space $(\Omega, \mathfrak{U}, P)$, and let $T_{n}$ denote the set of stop rules for $X_{1}, \ldots, X_{n}$. The "prophet" inequality $E\left(\max \left\{X_{1}, \ldots, X_{n}\right\}\right) \leqq k \sup \left\{E X_{t}: t \in T_{n}\right\}$ has been studied in the theory of semiamarts (e.g., [2-5]). Krengel and Sucheston [3] discovered that $2 \leqq k \leqq 4$ for all $n$ and all $X_{1}, \ldots, X_{n}$, and Garling's proof ([3], p. 237) shows that $k=2$, and that 2 is the best possible bound.

The purpose of this note is to offer a constructive proof that $k=2$, using extremal random variables called "long shots", and to show that in fact strict inequality holds in all non-trivial situations. The main result is

Theorem 1. Let $n>1$, and $X_{1}, X_{2}, \ldots, X_{n}$ be independent non-negative random variables with positive finite expectations. Then $E\left(\max \left\{X_{1}, \ldots, X_{n}\right\}\right)<2 \sup \left\{E X_{i}\right.$ : $\left.t \in T_{n}\right\}$.

\section{§. Proof of Theorem 1}

Throughout this section, all random variables are assumed to be non-negative with positive finite expectations. $E X$ will denote the expectation of $X, X \vee Y$ the maximum of $X$ and $Y,(X-Y)^{+}$the positive part $((X-Y) \vee 0)$ of $X-Y$, $V\left(X_{1}, \ldots, X_{n}\right)=\sup \left\{E X_{i}: t \in T_{n}\right\}$, and 


$$
R\left(X_{1}, \ldots, X_{n}\right)=E\left(X_{1} \vee \ldots \vee X_{n}\right) / V\left(X_{1}, \ldots, X_{n}\right) .
$$

With this notation, the conclusion of Theorem 1 is that $R\left(X_{1}, \ldots, X_{n}\right)<2$ for all $n>1$ and all $X_{1}, \ldots, X_{n}$.

Essential in the construction to follow is the notion of a "long shot", a twovalued random variable which is nearly always zero, but is very large on a set of small probability.

Definition. A long shot is a random variable $L$ defined by $L=0$ with probability $1-p$ and $=\mu$ with probability $p$, where $\mu>10^{6}$ and $0<p<10^{-6}$. (Any "large" and "small" constants will do.)

Lemma 1. Given $n>2$ and independent random variables $X_{1}, \ldots, X_{n}$ there exists a long shot $L$ satisfying $R\left(\lambda, X_{2}, \ldots, X_{n-2}, L\right)>R\left(X_{1}, \ldots, X_{n}\right)$, where $\lambda$ $=V\left(X_{2}, \ldots, X_{n}\right)$.

Since Lemma 1 reduces the number of random variables by one and since

$$
R\left(X_{1}, X_{2}\right)=E\left(X_{1} \vee X_{2}\right) / V\left(X_{1}, X_{2}\right)<\left(E X_{1}+E X_{2}\right) / \max \left\{E X_{1}, E X_{2}\right\} \leqq 2,
$$

the proof of Theorem 1 will be complete once Lemma 1 is established.

Proof of Lemma 1. First it is shown that $X_{1}$ may be replaced by the constant $\lambda$ $=V\left(X_{2}, \ldots, X_{n}\right)$, that is,

$$
\begin{aligned}
& R\left(X_{1}, \ldots, X_{n}\right) \\
& \leqq \\
& \quad\left[E\left(\lambda \vee X_{2} \vee \ldots \vee X_{n}\right)+E\left(X_{1}-\lambda\right)^{+}\right] / V\left(X_{1}, \ldots, X_{n}\right) \\
& \quad=\left[E\left(\lambda \vee X_{2} \vee \ldots \vee X_{n}\right)+E\left(X_{1}-\lambda\right)^{+}\right] /\left[V\left(\lambda, X_{2}, \ldots, X_{n}\right)+E\left(X_{1}-\lambda\right)^{+}\right] \\
& \quad \leqq R\left(\lambda, X_{2}, \ldots, X_{n}\right) .
\end{aligned}
$$

The first inequality in (1) follows since

$$
\begin{aligned}
E\left(X_{1} \vee \ldots \vee X_{n}\right) & \leqq E\left(X_{1} \vee \lambda \vee X_{2} \vee \ldots \vee X_{n}\right) \\
& =E\left(\lambda \vee X_{2} \vee \ldots \vee X_{n}\right)+E\left(X_{1}-\lambda \vee X_{2} \vee \ldots \vee X_{n}\right)^{+} \\
& \leqq E\left(\lambda \vee X_{2} \vee \ldots \vee X_{n}\right)+E\left(X_{1}-\lambda\right)^{+} ;
\end{aligned}
$$

the equality in (1) since (as an easy consequence of [1], p. 50) $V\left(X_{1}, \ldots, X_{n}\right)$ $=V\left(X_{2}, \ldots, X_{n}\right)+E\left(X_{1}-\lambda\right)^{+}$and $V\left(\mu, X_{2}, \ldots, X_{n}\right)=V\left(X_{2}, \ldots, X_{n}\right)$; and the last inequality since $0<V\left(\lambda, X_{2}, \ldots, X_{n}\right) \leqq E\left(\lambda \vee X_{2} \vee \ldots \vee X_{n}\right)$ and since $(a+\delta) /(b$ $+\delta) \leqq a / b$ for $a \geqq b>0$ and $\delta \geqq 0$.

Next, it will be shown that the last two random variables $X_{n-1}$ and $X_{n}$ may be replaced by some long shot $L$. Let $L_{p}$ be a long shot independent of $X_{2}, \ldots, X_{n-2}$ with $P\left(L_{p}=V\left(X_{n-1}, X_{n}\right) / p\right)=p>0$. Clearly $V\left(\lambda, X_{2}, \ldots, X_{n-2}, L_{p}\right)$ $=V\left(\lambda, X_{2}, \ldots, X_{n}\right)$. As $p \succ 0$,

$$
\begin{aligned}
E\left(\lambda \vee X_{2} \vee \ldots \vee X_{n-2} \vee L_{p}\right) \nearrow E\left(\lambda \vee X_{2} \vee \ldots \vee X_{n-2}\right)+E L_{p} \\
\quad=E\left(\lambda \vee X_{2} \vee \ldots \vee X_{n-2}\right)+E X_{n}+E\left(X_{n-1}-E X_{n}\right)^{+} \\
\quad \geqq E\left(\lambda \vee X_{2} \vee \ldots \vee X_{n-2}\right)+E X_{n}+E\left(X_{n-1}-\lambda \vee X_{2} \vee \ldots \vee X_{n-2}\right)^{+} \\
\quad=E\left(\lambda \vee X_{2} \vee \ldots \vee X_{n-1}\right)+E X_{n}>E\left(\lambda \vee X_{2} \vee \ldots \vee X_{n}\right) .
\end{aligned}
$$


Thus for $p^{\prime}$ sufficiently small, the long shot $L=L_{p^{\prime}}$ satisfies

$$
R\left(\lambda, X_{2}, \ldots, X_{n-2}, L\right)>R\left(\lambda, X_{2}, \ldots, X_{n}\right),
$$

which, with (1), completes the proof of the lemma.

\section{§3. Remarks}

An easy consequence of Theorem 1 is the result of Garling for infinite sequences:

Corollary 1. Let $X_{1}, X_{2}, \ldots$ be independent non-negative random variables. Then $E\left(X_{1} \vee X_{2} \vee \ldots\right) \leqq 2 V\left(X_{1}, X_{2}, \ldots\right)$.

If the independence assumption is dropped, the proportionate advantage a prophet enjoys over a gambler in an $n$-step game is at most $n$.

Proposition 1. If $X_{1}, X_{2}, \ldots, X_{n}$ are non-negative, then $E\left(X_{1} \vee \ldots \vee X_{n}\right)$ $\leqq n V\left(X_{1}, \ldots, X_{n}\right)$, and the bound $n$ is sharp.

Proof. Since $E\left(X_{1} \vee \ldots \vee X_{n}\right) \leqq E X_{1}+\ldots+E X_{n}$ and $V\left(X_{1}, \ldots, X_{n}\right)$ $\geqq \max \left\{E X_{1}, \ldots, E X_{n}\right\}$ it follows that $E\left(X_{1} \vee \ldots \vee X_{n}\right) \leqq n V\left(X_{1}, \ldots, X_{n}\right)$. For $n$ $=1, E X_{1}=V\left(X_{1}\right)$. To show that the bound $n$ is sharp for $n>1$, let $p \in(0,1)$ be given and define random variables $X_{1}, \ldots, X_{n}$ jointly by $P\left[\left(X_{1}, \ldots, X_{n}\right)\right.$ $\left.=\left(p^{0}, p^{-1}, \ldots, p^{-j}, 0, \ldots, 0\right)\right]=p^{j}-p^{j+1}$ if $0 \leqq j \leqq n-2$, and $=p^{n-1}$ for $j=n-1$. Then $X_{1}, \ldots, X_{n}$ is a martingale and $V\left(X_{1}, \ldots, X_{n}\right)=E X_{1}=1$. Observe that $E\left(X_{1} \vee \ldots \vee X_{n}\right)=(n-1)(1-p)+1$, and let $p \searrow 0$.

If one drops the non-negativity assumption, on the other hand, the prophet's proportionate advantage may be arbitrarily high in even a 2-step game of independent random variables.

Example 1. Fix $M \gg 0$. Let $X_{1} \equiv 1$, and define $X_{2}$ by $P\left(X_{2}=2 M\right)=P\left(X_{2}=\right.$ $-2 M)=1 / 2$. Then $V\left(X_{1}, X_{2}\right)=1$, and $E\left(X_{1} \vee X_{2}\right)=M+1 / 2$.

Acknowledgement. The authors would like to thank U. Krengel for several conversations and private correspondence including unpublished notes and manuscripts.

\section{Bibliography}

1. Chow, Y.S., Robbins, H., Siegmund, D.: Great Expectations: The Theory of Optimal Stopping. Boston: Houghton Mifflin 1971

2. Edgar, G.A., Sucheston, L.: Amarts; A Class of Asymptotic Martingales. A. Discrete Parameter. J. Multivariate Analysis, 3, 193-221 (1976)

3. Krengel, U., Sucheston, L.: Semiamarts and Finite Values. Bulletin of the Amer. Math. Soc. 83, 745-747 (1977)

4. Krengel, U., Sucheston, L.: On Semiamarts, Amarts, and Processes with Finite Value in Probability on Banach Spaces. New York: Marcel Dekker 1978

5. Krengel, U., Sucheston, L.: How to Bet Against a Prophet. (Some $L^{1}$ Dominated Estimates for Semiamarts). Abstract, Notices of the Amer. Math. Soc. 24, A-159 (1977) 